\title{
Case Report of Posttraumatic Middle Cerebral Artery Territory Infarct
}

\author{
Ajaya Kumar Ayyappan Unnithan ${ }^{1}$ \\ ${ }^{1}$ Mar Gregorios Memorial Muthoot Medical Centre, Kozhencherry, \\ Kerala, India \\ Indian J Neurosurg 2017;6:50-54.

\begin{abstract}
Address for correspondence Ajaya Kumar Ayyappan Unnithan, MBBS, MS, DNB, MCh, Muthoot Healthcare, Kozhencherry, Pathanamthitta, Kerala 689641, India (e-mail: drajayakumara@gmail.com).
\end{abstract}

\author{
Abstract \\ Keywords \\ - diffuse brain injury \\ - posttraumatic \\ cerebral infarction \\ - middle cerebral \\ artery territory \\ infarction \\ - decompressive \\ craniectomy \\ - subdural hygroma \\ - burr hole \\ - hydrocephalus \\ - VP shunt \\ - syndrome of \\ trephined \\ - cranioplasty
}

A 27-year-old man had severe diffuse brain injury. The patient developed malignant right MCA territory infarction on second day. Emergency decompressive craniectomy was done. He was ventilated. He developed subdural hygroma on the opposite side that was drained. He improved slowly. He had hydrocephalus. VP shunt was done. He became conscious but dependent. PEG was done for feeding. Cranioplasty was done for syndrome of trephined. The patent improved to a state of good cognition with residual motor aphasia and left hemiplegia.

Posttraumatic cerebral infarction is an indicator of poor prognosis. Vasospasm, intimal dissection, and thrombosis are the mechanisms. MCA territorial infarction is rare. Usual mechanism is impact of rotational forces on relatively fixed M1 segment on the posterior margin of lower wing of sphenoid, resulting in arterial dissection or intimal damage.

\section{Introduction}

Posttraumatic cerebral infarction indicates poor clinical outcome in head injury. Middle cerebral artery (MCA) territory infarction is rare and is caused by stretching and attenuation of MCA and increased intracranial pressure (ICP). This is a case report of a young patient who had posttraumatic MCA territory infarction.

\section{Case Report}

A 27-year-old man was brought to emergency services following road traffic accident. The patient was riding pillion on a bike, and the driver died in casualty due to head injury and pelvic fracture. He was in unconscious state with the Glasgow Coma Scale (GCS) of E1M3V1. The pupils were sluggishly reacting to light bilaterally. He was intubated and ventilated. Computed tomography (CT) of the head showed diffuse injury-subarachnoid hemorrhage, multiple small contusions, right MCA hyperdensity, right temporofrontal hypodensity, ventricular effacement, and small midbrain contusion.

Antiedema and cerebral protectant therapy and hyperventilation were started and, nimodipine and citicoline were given. The next day he worsened with GCS E1M1 and dilated pupil on the right side, and repeat CT of the brain showed right (MCA) territory infarct with mass effect and midline shift (-Fig. 1). Contrast-enhanced CT showed nonvisualization distal to M1 (-Fig. 2).

Emergency right frontotemporoparietal decompressive craniectomy and infarct decompression were done. Duraplasty was done with G patch. The brain was very tense (- Fig. 3). The patient had GCS of E2M2 postoperatively and CT of the brain received

July 28, 2015

accepted

April 4, 2016

published online

September 1, 2016
DOI http://dx.doi.org/

10.1055/s-0036-1584594. ISSN 2277-954X. (c) 2017 Thieme Medical and Scientific

Publishers Private Ltd.
License terms

()(1) $\Theta \circledast$ 


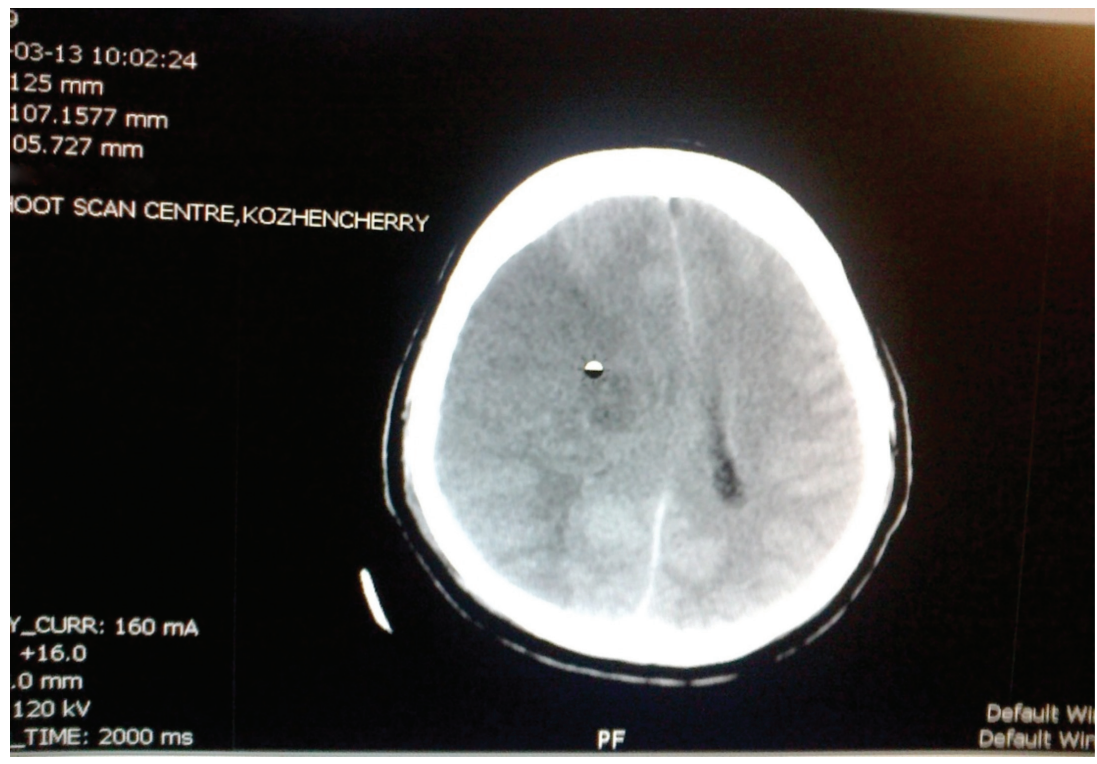

Fig. 1 CT of the brain showing right MCA territory infarct with mass effect and midline shift.

showed persisting edema and cerebral herniation into defect. He was given mannitol, nimodipine, and thiopentone infusion, and paralyzed controlled hyperventilation.

Chest X-ray on 7th day showed early changes of ventilatorassociated pneumonia. Tracheostomy was done for tracheobronchial toilet. CT of the brain after 1 month showed subdural hygroma on the left side. Burr hole drainage was done (-Fig. 4).

The patient showed signs of improvement. He became alert with eye opening with command and had M3 movement in the right upper limb on pain and attempted to move right lower limb on command at 1 month and 5 days. Magnetic resonance imaging (MRI) showed damage to the right temporoparietal lobes (-Fig. 5). The patient became conscious, had spontaneous eye opening, and could obey commands by 2 months. He was completely weaned off ventilator to T-piece with oxygen support.

At 3 months, he had dulling of sensorium and CT of the brain showed hydrocephalus (-Fig. 6). Ventriculoperitoneal shunt was done. Cerebrospinal fluid (CSF) came under high

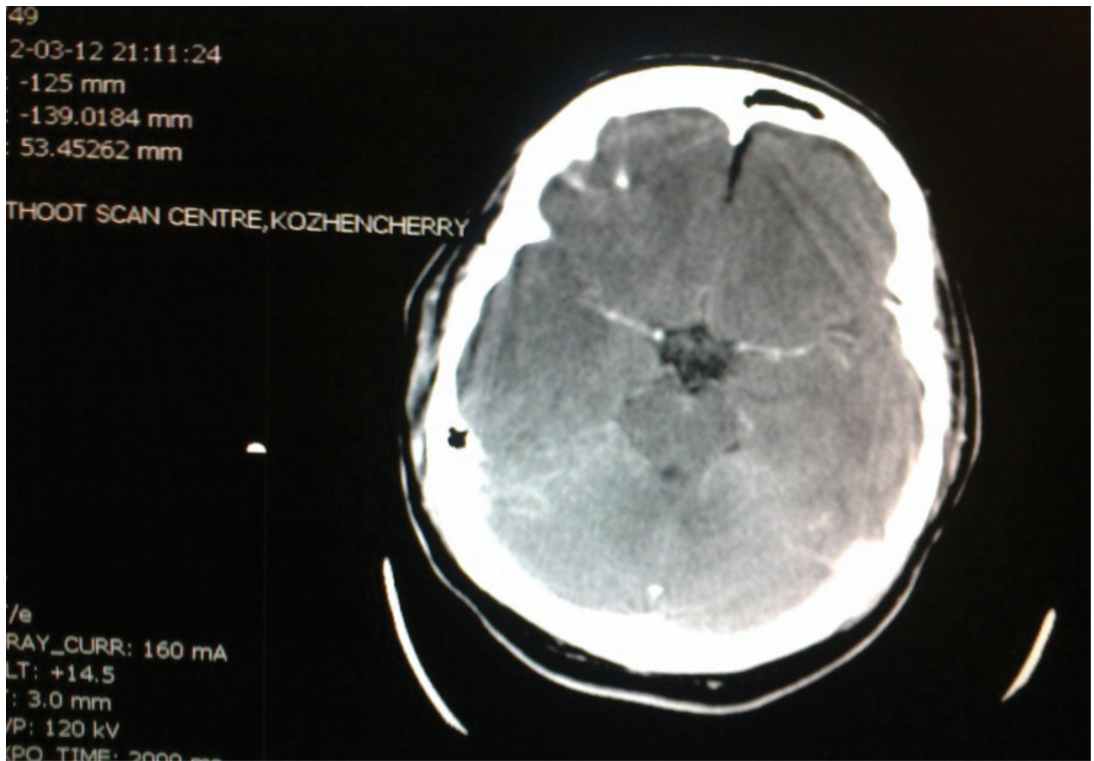

Fig. 2 Contrast-enhanced CT shows nonvisualization distal to M1. 


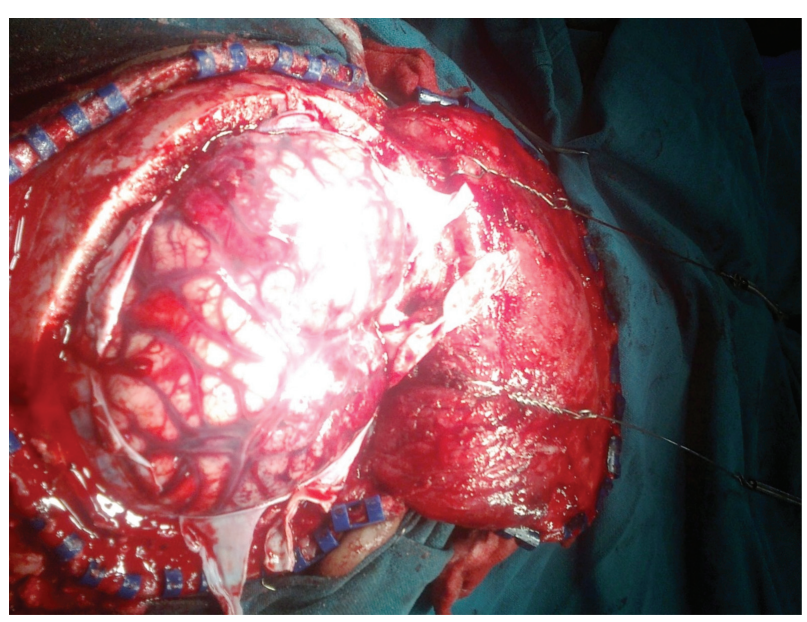

Fig. 3 Peroperative photograph showing tense brain.

pressure. Postoperative CT showed relaxed brain. By 4 months, the patient was alert and could obey commands with improved swallowing capability. He could tolerate removal of tracheostomy tube at 5 months and was on oxygen by mask. He could take oral liquids by mouth without cough and could communicate by gestures by 5 months. Percutaneous endoscopic gastrostomy was done to reduce aspiration.

The patient had syndrome of trephined at 14 months, viz. dulling of sensorium, decreased response, and eye opening. CT of the brain showed severely sunken brain (-Fig. 7). Cranioplasty was done. The bone was harvested from the abdomen, and the bone had undergone resorption. Titanium mesh was inserted (-Fig. 8). Postcranioplasty CT of the brain was satisfactory. At present the patient is conscious, reads, writes, shows gestures, and vocalizes words. Left hemiplegia is persisting.

\section{Discussion}

Types of diffuse brain injury include diffuse vascular injury, diffuse axonal injury, diffuse ischemic brain damage, and diffuse brain swelling. ${ }^{1}$ Diffuse vascular injury is most serious and found in patients who succumb soon after head injury. Damage to the medium and large blood vessels occurs. Internal carotids and vertebral arteries and branches can be involved. It can produce intimal tear, vasospasm, pseudoaneurysm, and infarction.

Frequency of posttraumatic cerebral infarction ranges from 1.9 to $10.4 \%{ }^{2}$ It is an indicator of poor clinical outcome and is associated with a high mortality rate, despite appropriate medical and surgical interventions. Mechanisms include cerebral vasospasm, vascular injury, embolization, and systemic hypoperfusion. Common infarctions include posterior cerebral artery (PCA) territory infarction in uncal herniation and anterior cerebral artery (ACA) territory infarction in subfalcine herniation.

MCA territory infarction is rare and occurs due to stretching and attenuation of the MCA, increased ICP, and direct pressure effect from an extra-axial hematoma. ${ }^{3}$ In case of MCA, mechanism in primary vascular injury is usually due to the impact of rotational forces on relatively fixed M1 segment on the posterior margin of lower wing of the sphenoid, resulting in arterial dissection or intimal damage and thus thrombosis. ${ }^{4}$

\section{Review of Literature}

Total 65 cases of traumatic MCA occlusion are discussed in the literature ${ }^{4} ; 18$ were fatal with a resulting mortality of $27.7 \%$. Review of the 18 autopsy cases demonstrates that subintimal dissection is the most likely cause of MCA occlusion, noted in 14 (78\%) of 18 cases. Two (11\%) cases involved thrombosis in the artery.

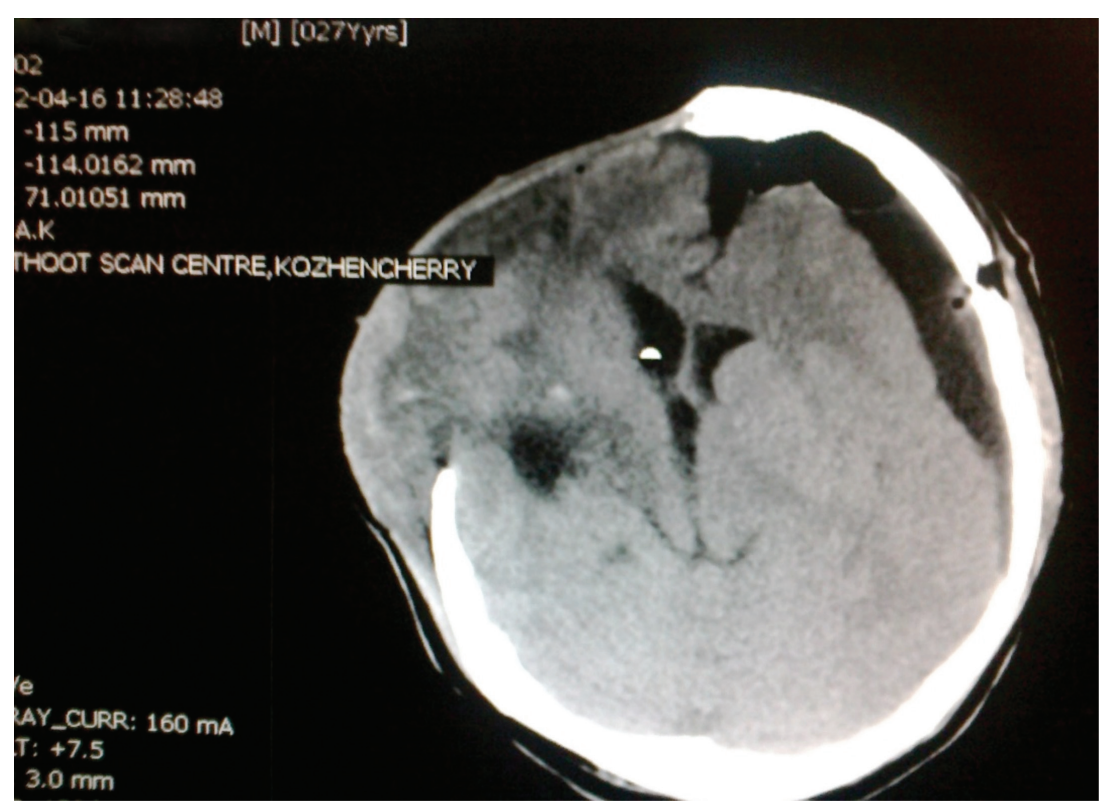

Fig. 4 Burr hole for left subdural hygroma. 


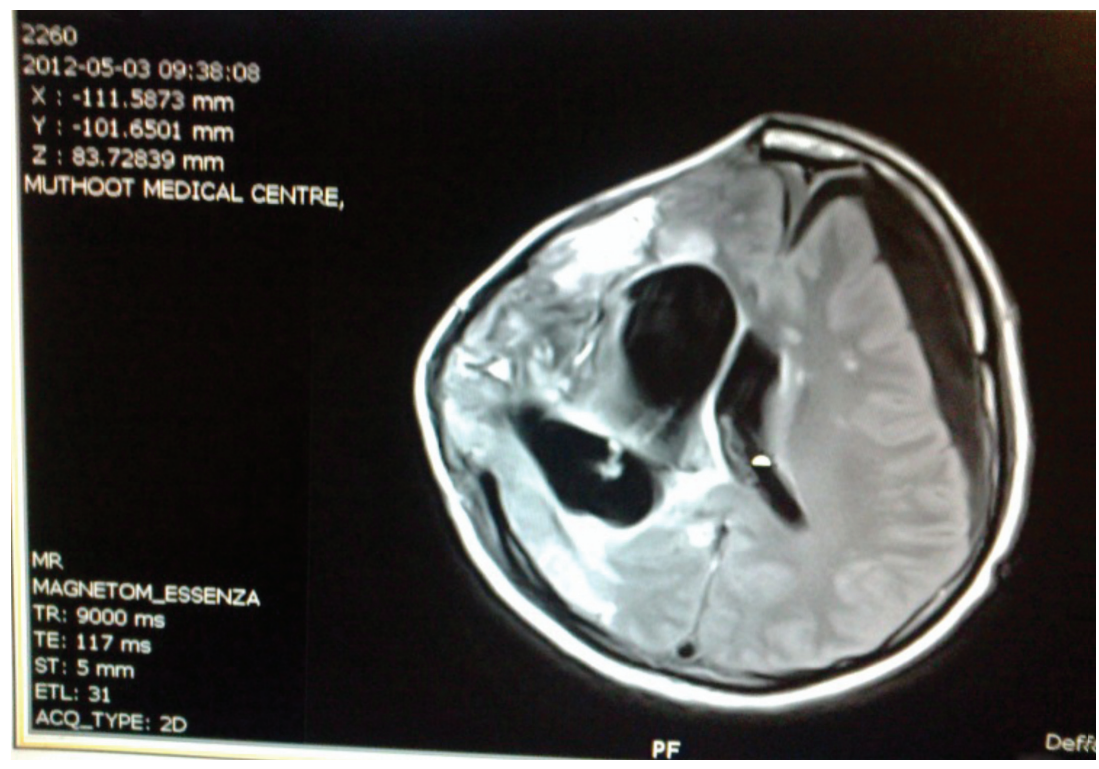

Fig. 5 MRI showing damage to the right temporoparietal lobes.

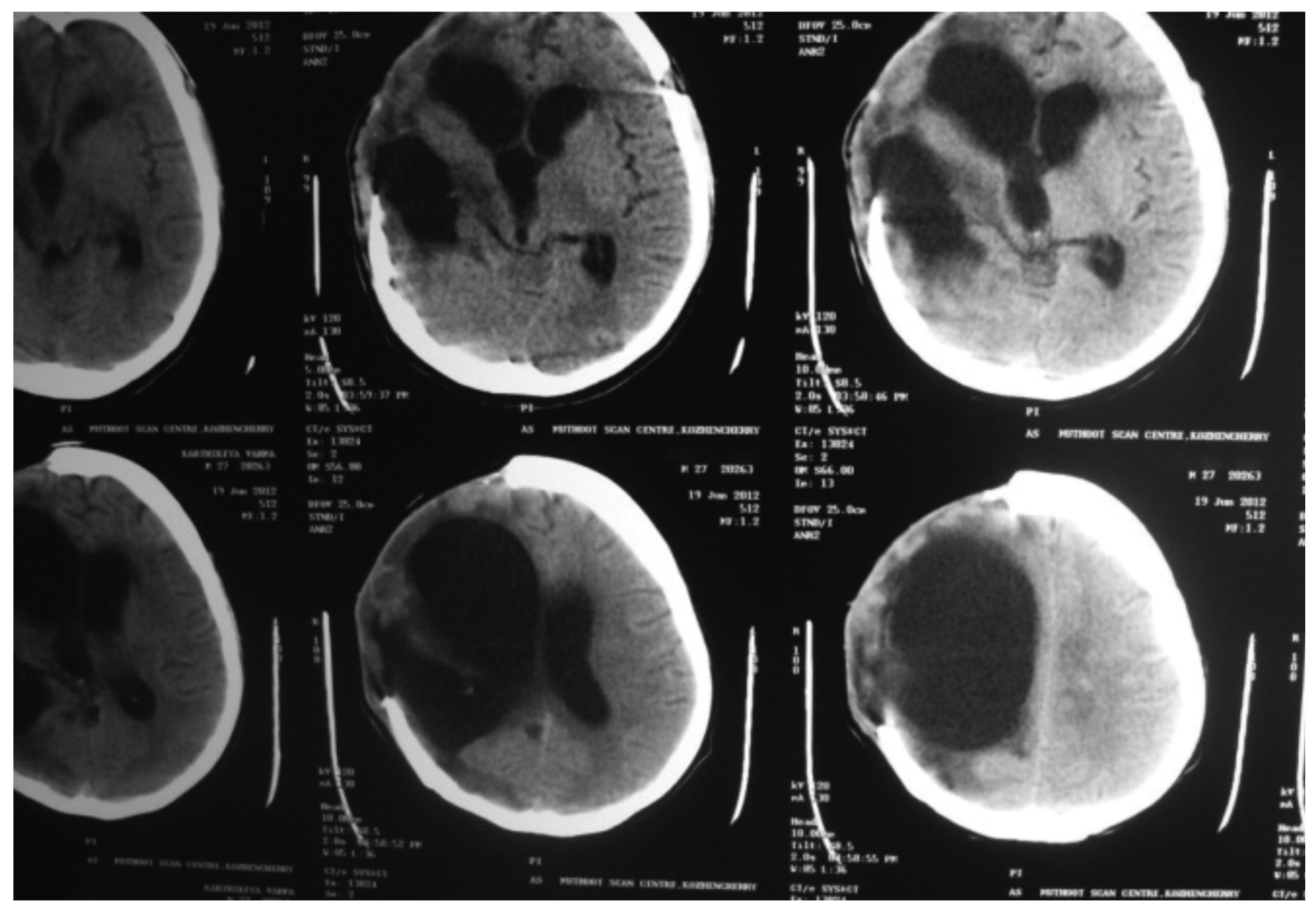

Fig. 6 CT showing hydrocephalus. 


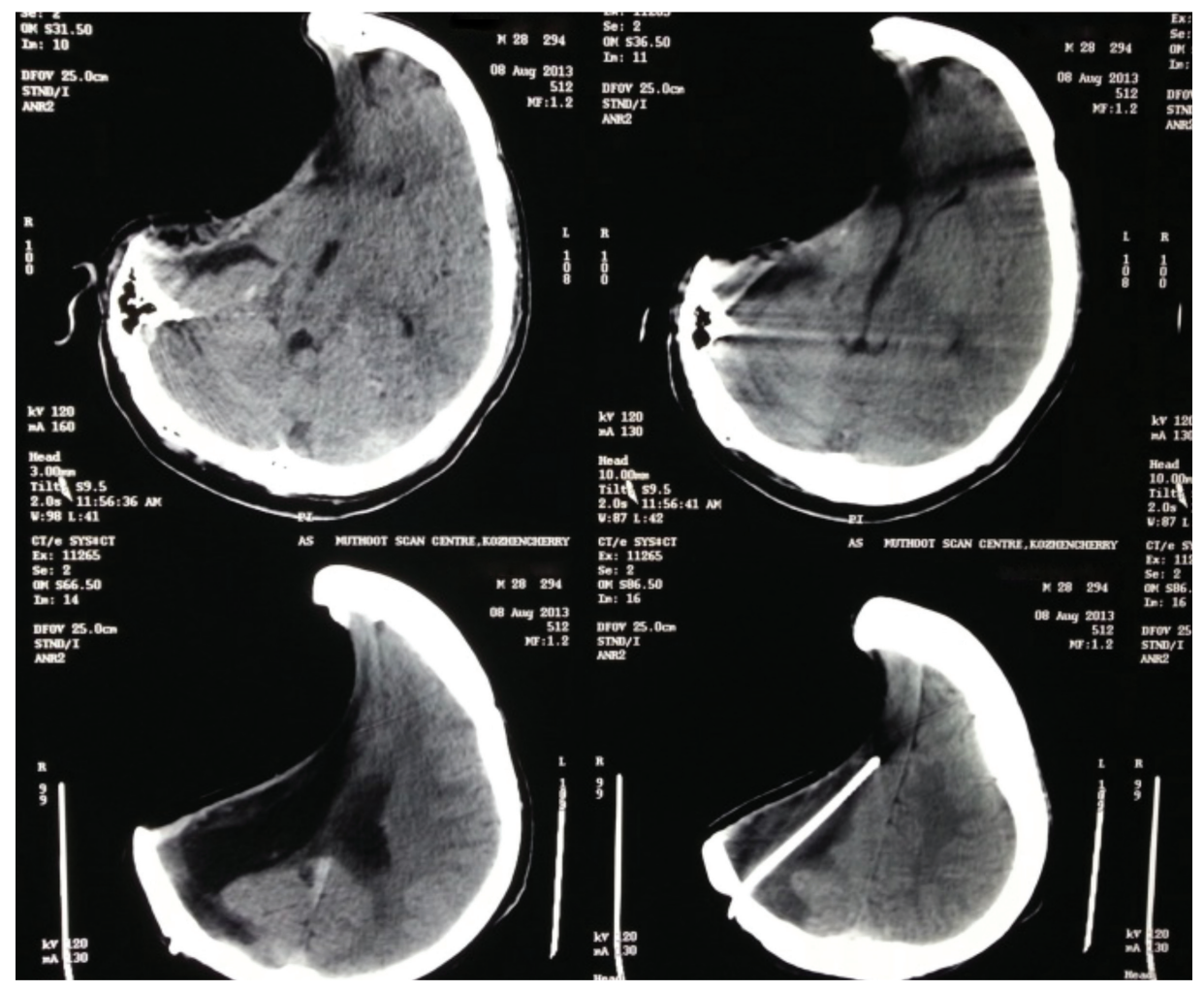

Fig. 7 CT showing sunken brain.

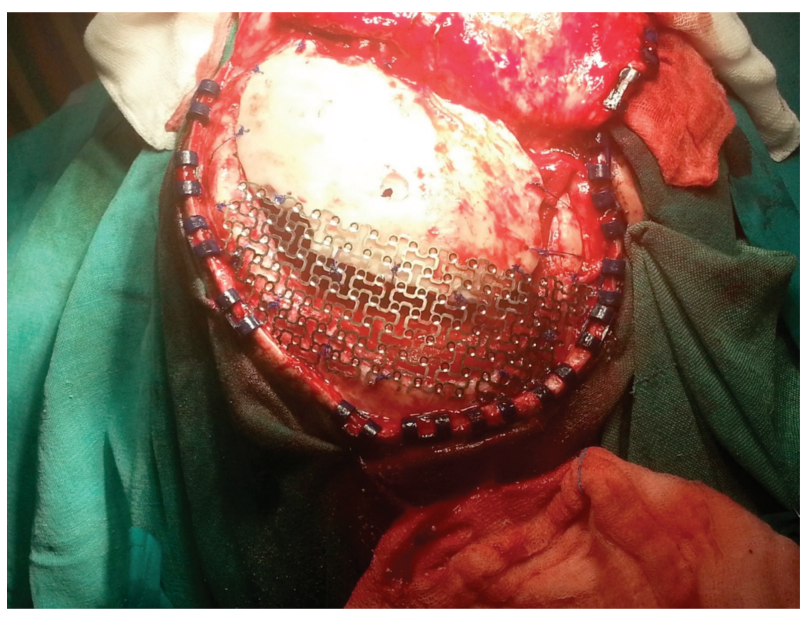

Fig. 8 Cranioplasty using bone and titanium mesh.

One surviving case of primary vascular injury was reported by Mobbs and Chandran (Department of Neuroscience, The Canberra Hospital, Australia). ${ }^{4}$ A 15 -year-old adolescent boy fell from a bicycle and had left hemiparesis on 12th hour. CT of the brain showed right MCA territory infarction. The patient recovered by medical management alone.

\section{Conclusion}

This is a case report of a young patient who survived severe diffuse injury with right MCA territory infarction through prolonged medical and surgical treatment and physiotherapy to acceptable level of cognition. Posttraumatic cerebral infarction is indicator of poor prognosis. Impact of rotational force on M1 segment against sphenoid is the mechanism considered.

\section{References}

1 Sankar SK, Mahadevan A. Ramamurthi \& Tandon's Textbook of Neurosurgery. Head Injury Pathology. 3rd ed. New Delhi, India: Jaypee Brothers Medical Publishers; 2012:319-341

2 Ham HY, Lee JK, Jang JW, Seo BR, Kim JH, Choi JW. Post-traumatic cerebral infarction: outcome after decompressive hemicraniectomy for the treatment of traumatic brain injury. J Korean Neurosurg Soc 2011;50(4):370-376

3 Wani AA, Babu ML, Altaf RU, et al. Post traumatic ischemic stroke in posterior and middle cerebral arteries following evacuation of extradural hematoma. J Paediatric Neurosci 2007;2:92-93

4 Mobbs RJ, Chandran KN. Traumatic middle cerebral artery occlusion: case report and review of pathogenesis. Neurol India 2001;49(2):158-161 